\title{
Impact Position Variability in Golfers of Differing Skill Level
}

\author{
Ben L. Langdown \\ PGA, National Training Academy, UK; University of Birmingham \\ Matthew W. Bridge and François-Xavier Li \\ University of Birmingham
}

\begin{abstract}
This study examined the variable error (VE) for the golf impact position of high and low skilled golfers. A consistent shot type was employed by 20 golfers from 2 skill groups over $10-15$ shots with a midiron. Analysis reported similar positional variability across categories. Significant differences were found for the whole group in VE of the distance of various body segments to the ball $(p<.001)$ where variability reduced across the distance of the ball to the midpoint of the stance, compared with the pelvis and shoulders. Alignment variability significantly decreased toward distal segments of the kinematic sequence with VE for the alignment of stance relative to pelvis compared with stance relative to shoulders and pelvis relative to shoulders showing reduced levels $(p<.001)$ in the low skilled golfers. Tilt variability in the frontal plane around the sagittal/anterior-posterior (AP) axis also presented significantly reduced levels from the pelvis to the shoulders $(p<.001)$ with no effect of skill level. Conclusions suggest that coaches should pay particular attention to the variability presented at the distal end of the kinematic sequence and the alignment of the stance in relation to the pelvis.
\end{abstract}

Keywords: impact, golf, variability

Most sports possess critical features that contribute to the successful performance of a skill (McPherson, 1990) and if modified too greatly these critical features can affect the outcome and can result in detriments to performance (Arend \& Higgins, 1976). In ball sports these features are often most prevalent at the moment of impact between either a sports implement or human performer and the ball. Critical impact factors at the instant of contact govern the parameters of ball launch and flight and subsequent shot outcome. In golf, the impact factors of angle of attack, centeredness of strike (impact location on the clubface), clubface alignment (in relation to the target line), path of the clubhead and clubhead velocity

\footnotetext{
Langdown is with the The Professional Golfers' Association, National Training Academy, Ping House, Sutton Coldfield, West Midlands, UK, and also the School of Sport, Exercise and Rehabilitation Sciences, University of Birmingham, Edgbaston, Birmingham, UK. Bridge and Li are with the School of Sport, Exercise and Rehabilitation Sciences, University of Birmingham, Edgbaston, Birmingham, UK.
} 
(Tuxen, 2009) present the areas in which coaches strive for reduced detrimental variability (Langdown, Bridge \& Li, 2012). Betzler, Monk, Wallace and Otto (2012) have shown that more highly skilled golfers exhibited significantly reduced shot variability in clubhead velocity, efficiency of strike (resultant ball speed as a direct result of clubhead speed: coefficient of restitution), centeredness of strike, angle of attack, club path, and face angle at impact compared with less skilled golfers. Reduced variability in factors governing movement outcome accuracy has also been found in table tennis (Bootsma \& van Wieringen, 1990) and basketball (Miller, 2002). Miller (2002) reported a decreasing level of movement variability moving from toe to ball in basketball free throw kinematics, suggesting that toward the accuracy dominated end of the kinematic sequence expert performers present reduced variability. The impact position of a golfer's body could follow this pattern with reduced kinematic variability in the body segments that have influence upon the accuracy of strike of the golf ball i.e., reduced variability from foot position through to the hand and clubhead positions (Langdown et al., 2012).

Behavior has been suggested to emerge from a system of constraints when performing a goal orientated task rather than a consistent motor program (Newell, 1986). This would suggest that movement variability to meet a task outcome will exist. Such variability may be detrimental or functional to the movement outcome and it is important for golf coaches to understand the role that movement variability plays within the swing. We have previously shown that address variability in alignment between the shoulders and stance is reduced for more highly skilled golfers compared with less skilled golfers (Langdown, Bridge \& $\mathrm{Li}$, in press). We also reported that distance, alignment and tilt variability decreased across proximal to distal body segments when addressing the golf ball. These findings emphasize the importance of reducing variability in the body segments that have an increasing effect on performance accuracy.

Coaches (e.g., Hebron, 2001; Mann, Griffin \& Yocom, 1998) suggest that the address positions a player adopts can influence impact with the ball. Mann et al. (1998) have suggested that the position of the ball has a critical influence over the angle of attack and that the body reacts in the attempt to "find" the ball with the clubhead, in fact they state that the ball position affects the swing more than any other alignment factor. As well as the angle of attack as suggested by Mann et al. (1998), there will also be other effects on factors such as club path and club face orientation at impact which will alter the resultant ball flight. Mann et al. (1998) continue by suggesting that compensations are important if the ball has been positioned poorly at address. This shows the importance of address and how variability here will have subsequent effects on the position of the body at impact as the golfer solves the movement problem of returning the club to the ball to attempt to meet the task goal. The problem of address variability and consequential impact position variability becomes increasingly difficult when moving away from the flat surfaces of a range bay or the tee boxes on the course; research is yet to establish how golfers cope with this environmental factor.

Limited research exists into the variability of body positions at the moment of clubhead impact with the golf ball. Horan, Evans and Kavanagh (2011) reported that both male and female golfers showed decreasing hand position variability from the top of the backswing through to ball contact even though they used different coordination strategies to zero in on impact. Clubhead trajectory was also shown 
to follow a similar funneling trend. Bradshaw et al. (2009) recently studied the kinematic variability that occurs within the swings of high vs. low handicapped golfers using 2D video analysis and found no differences between skill levels for the variability presented in the trunk, lead wrist and elbow angles at impact. However, it must be remembered that a true representation of the movement of the swing is only achievable through 3D kinematic analysis. With suggestions that the impact factors form the critical features of golf swing performance (Langdown et al., 2012) it is crucial that the movements of the body are also analyzed to see if these are affected by skill level. The five impact factors are all influenced by the position the golfer sets at address to the golf ball and movements the golfer makes during the downswing which emerge from the environmental, organism and task constraints. However, it is suggested (Cochran \& Stobbs, 1968) that if the golfer needs to make movement adjustments to the swing this has to be done within the backswing as the duration of the downswing to impact is too short. This notion is supported by research (e.g., Verbruggen \& Logan, 2009) suggesting that typically, participants can inhibit or change a specific response when a stop signal is presented close to the moment of stimulus presentation (in this case the beginning of the swing), but they cannot inhibit or change their movement when the stop signal is presented close to the moment of response execution (i.e., the downswing moving toward impact). Other sports have shown that there is a "zeroing in" or "homing in" process that allows variability to be minimized toward the critical phase of performance (e.g., impact) (e.g., table tennis: Bootsma \& van Wieringen, 1990; long jump approach phase: Lee, Lishman \& Thomson, 1982; Scott, Li, \& Davids, 1997).

This study aims to inform coaches of how a golfer's ability level may influence variability in impact positions and its changing magnitude in the proximal to distal segments of the golfer's body at the point of impact between the clubhead and the golf ball. It is hypothesized that more highly skilled golfers will present reduced variability at impact compared with less skilled golfers across body position variables. If the critical features of the golf shot with a midiron are the impact factors then this would suggest that with increasing skill level will come increasing proficiency at presenting an impact position that maximizes distance and accuracy toward a given goal. This hypothesis has also been founded on elite players in other sports exhibiting a funneling or "zeroing in" of movement toward impact in the sports of table tennis (Bootsma \& Van Wieringen, 1990) and long jump approach run (Lee et al., 1982; Scott et al., 1997).

\section{Method}

\section{Participants}

20 golfers (17 male and 3 female) were randomly recruited following local committee ethics approval and formed high and low handicap groups which included golfers between the ages of 18 years and 27 years $(M \pm S D$ : HSG $=21 \pm 1 \mathrm{yr}$ and $\mathrm{LSG}=21 \pm 2 \mathrm{yr}$ ). Participants were categorized by CONGU handicap (high (HSG): $n=10$ Category 1 : handicaps up to $5.4 \&$ professionals with a mean clubhead speed of $39.2 \mathrm{~m} / \mathrm{s}$. Low (LSG) $n=10$ Category 3: handicaps of 12.5-20.4 with a mean clubhead speed of $37.2 \mathrm{~m} / \mathrm{s}$ ). This method of categorization is common practice 
within golf research but does not identify areas of specific skill that contributes to a lower handicap. It merely represents each individual's overall skill levels relative to their home course. All participants reported that they were injury free and experienced no pain during their golf swing.

\section{Procedure}

All participants performed 10-15 swings with their own mid iron (6 or 7 iron) that were captured at $250 \mathrm{~Hz}$ using a 13 camera VICON MX system and 34 reflective markers. All participants were asked to address the ball and perform their normal swing to produce a consistent ball flight with maximum accuracy and distance toward an identified target on a golf net. For a full description of the experimental procedure see Langdown, Bridge and Li (2013).

\section{Selection of Variables}

Variables were selected based on address variables presented in Langdown et al. (2013) where a panel of expert coaches from the Professional Golfers' Association of Great Britain and Ireland membership were consulted on the importance of the consistency of a number of variables that measured a golfer's position at address. It was important to see the influence of these positions at impact to assess the effect of various body segments in relation to the critical moment in the swing. The following variables (Table 1) were selected to be measured.

\section{Table 1 Static Variables Measured at Impact Position}

\begin{tabular}{ll}
\hline Variable & Definition \\
\hline Distance of Stance to Ball & $\begin{array}{l}\text { True distance to the ball from the middle of the stance (deter- } \\
\text { mined by the midpoint of the vector between the 1st toe of } \\
\text { each foot). }\end{array}$ \\
Distance of Pelvis to Ball & $\begin{array}{l}\text { True distance of the center of the pelvis to the ball (center } \\
\text { pelvis was taken as the midpoint of the vector between the left } \\
\text { and right ASIS). }\end{array}$ \\
Distance of Shoulder to Ball & $\begin{array}{l}\text { True distance of the center of the shoulder line to the ball } \\
\text { (center of the shoulder line was taken as the midpoint of the } \\
\text { transverse plane projection of the vector between the left and } \\
\text { right acromion processes). }\end{array}$ \\
& $\begin{array}{l}\text { Hip tilt in the frontal plane around the sagittal/anterior-poste- } \\
\text { rior (AP) axis (determined using the tangent of the transverse } \\
\text { plane projection of the vector between the left and right ASIS). }\end{array}$ \\
Pelvic Tilt & $\begin{array}{l}\text { Shoulder tilt in the frontal plane around the sagittal/anterior- } \\
\text { posterior (AP) axis (determined using the tangent of the trans- } \\
\text { verse plane projection of the vector between the left and right } \\
\text { acromion processes). }\end{array}$ \\
Shoulder Tilt & $\begin{array}{l}\text { Angle of the pelvis in relation to the stance (open or closed). } \\
\text { Angle of the shoulders in relation to the stance (open or closed). }\end{array}$ \\
Stance to Pelvis Alignment & Angle of the shoulders in relation to the pelvis (open or closed). \\
\hline Stance to Shoulder Alignment \\
Pelvis to Shoulder Alignment
\end{tabular}


Swing Processing. After reconstruction and labeling of marker trajectories, each individual's swing raw data files were exported from the VICON Nexus system as a comma separated values file. Functions and scripts were written for data processing in Excel and allowed impact to be identified through an average of 2 frames ( 1 prior and 1 post impact) or a single frame (where the clubhead was in contact with the ball) depending upon captured positions of the clubhead. Positional data analysis allowed all variables to be measured at impact over the series of shots played. Where markers were not visible at impact the trials were removed from analysis.

\section{Data Analysis}

All data were checked for approximation to the normal distribution, and group and individual means and standard deviations were calculated. Natural log transformations were used to normalize for shoulder tilt, stance to pelvis alignment, and pelvis to shoulder alignment variable error, log to base 10 for pelvis and shoulder distance to ball variable error and a reciprocal transformation for stance to shoulder alignment variable error data. To assess the variability between trials (within golfer) and between levels of skill (HSG compared with LSG) variable error measures were used (Schmidt \& Lee, 2005) where Variable Error $(\mathrm{VE})=\left(\sqrt{ } \sum\left(x_{i}-\mathrm{M}\right)^{2} / n\right)$ and $x_{i}$ is the value of the selected variable for each trial, $\mathrm{M}$ is the mean average of this variable for all shots played and $n$ is the number of shots played. Mixed factorial MANOVAs (repeated factor: VE, group factor: skill level) were used to assess any differences in VE between variable groups of distance (stance, pelvis and shoulder midpoints to ball), alignment (pelvis-stance; shoulder-stance; shoulder-pelvis) and tilt (pelvis; shoulder). When significant effects were observed post hoc tests with a Bonferroni correction $(\mathrm{a}=0.016)$ were used to identify where differences existed between measures. All data analysis was carried out using SPSS 20.0 and data are reported as mean and standard deviation unless otherwise stated.

\section{Results}

There was a significant main effect across the whole group in VE (Table 2) of the distance (Wilks's $\gamma=0.33, F(2,17)=17.5, p<.001, \eta p^{2}=0.67$ ), alignment (Wilks's $\Lambda$ $\left.=0.43, F(2,17)=11.21, p<.001, \eta p^{2}=0.569\right)$ and tilt $($ Wilks's $\gamma=0.42, F(1,18)=24.5$, $\left.p<.001, \eta p^{2}=0.58\right)$ variable groups.

\section{Distance VE}

A significant interaction was found between skill categories and distance VE (Wilks's $\Lambda=0.611, F(2,17)=5.413, p=.015, \eta p^{2}=0.389$ ) showing that overall variability was different between groups. However, Bonferroni post hoc comparisons presented no differences between groups in any of the separate distance parameter variable errors (stance: $p=.351$; pelvis: $p=.273$; shoulders: $p=.295$ ). Distance VE of the stance to ball exhibited the most variability in this group with the distance VE of both the pelvis to ball and shoulder to ball being approximately $68 \%$ of its value (Table 2). When VE was considered within each category of skill the same pattern was found: Stance distance VE was significantly greater than both pelvis $(\mathrm{HSG}=p=.035 ; \mathrm{LSG}=p<.001)$ and shoulder distance $(\mathrm{HSG}=p=.015 ; \mathrm{LSG}=$ 
Table 2 Mean $\pm S D$ for Variable Error (VE) for Each Variable.

\begin{tabular}{lccc}
\hline Impact variable & $\begin{array}{c}\text { Whole } \\
\text { group VE }\end{array}$ & $\begin{array}{c}\text { High skill } \\
\text { group VE }\end{array}$ & $\begin{array}{c}\text { Low skill } \\
\text { group VE }\end{array}$ \\
\hline Distance of Stance to Ball $(\mathrm{mm})$ & $11.01 \pm 3.05^{\#}$ & $10.23 \pm 2.48$ & $11.80 \pm 3.48$ \\
Distance of Pelvis to Ball $(\mathrm{mm})$ & $7.55 \pm 1.91^{\#}$ & $8.02 \pm 1.97$ & $7.08 \pm 1.82$ \\
Distance of Shoulder to Ball $(\mathrm{mm})$ & $7.52 \pm 2.31^{\#}$ & $6.97 \pm 1.79$ & $8.06 \pm 2.73$ \\
Pelvis Tilt $\left({ }^{\circ}\right)$ & $1.23 \pm 0.49^{£}$ & $1.26 \pm 0.47$ & $1.21 \pm 0.54$ \\
Shoulder Tilt $\left({ }^{\circ}\right)$ & $1.09 \pm 0.36^{£}$ & $0.96 \pm 0.22$ & $1.22 \pm 0.44$ \\
Pelvis to Stance Alignment $\left({ }^{\circ}\right)$ & $2.31 \pm 0.93^{\$}$ & $1.92 \pm 0.43$ & $2.69 \pm 1.14$ \\
Shoulder to Stance Alignment $\left(^{\circ}\right)$ & $1.87 \pm 0.83^{\$}$ & $1.67 \pm 0.66$ & $2.08 \pm 0.97$ \\
Shoulder to Pelvis Alignment $\left({ }^{\circ}\right)$ & $1.85 \pm 0.98^{\$}$ & $1.92 \pm 0.83$ & $1.78 \pm 1.16$ \\
\hline
\end{tabular}

Note ${ }^{\# 1}$ indicate significant differences between variables $(\mathrm{p}<0.05)$.

$p=.017) \mathrm{VE}$ but there was no significant difference between pelvis and shoulder distance VE $(\mathrm{HSG}=p=.227 ; \mathrm{LSG}=p=.404)$.

\section{Alignment VE}

A significant interaction was found between skill categories and alignment relationships VE (Wilks's $\Lambda=0.57, F(2,17)=6.38, p=.009, \eta p^{2}=0.43$ ) showing that overall variability was different. Again, Bonferroni post hoc comparisons presented no VE differences between skill categories in any of the separate alignment relationship variables (stance to pelvis alignment relationship: $p=.069$; pelvis to shoulders: $p$ $=.626$; stance to shoulders: $p=.274$, Table 2). The largest alignment VE exhibited in this group was between stance and pelvis with both the alignment VE of the pelvis to shoulders and stance to shoulders being approximately $80 \%$ of its value (Table 2). When VE was considered within each category of skill, differences were found for LSG: a reduction in VE was found between stance to shoulder alignment compared with stance to pelvis alignment $(p=.007$, Table 2$)$ and between pelvis to shoulder alignment compared with stance to pelvis alignment $(p<.001)$. HSG presented no significant differences between all alignment variables.

\section{Tilt VE}

VE was significantly reduced in shoulder tilt compare with pelvic tilt at impact (Wilks's $\Lambda=0.42, F(1,18)=24.5, p<.001, \eta p^{2}=0.58$ ). There was no interaction effect of group (Wilks's $\Lambda=0.92, F(1,18)=1.57, p=.23, \eta p^{2}=0.08$ ) with both HSG and LSG showing similar VE levels in each segment.

\section{Discussion}

This study has examined whether there were differences in the variability of specified body impact position parameters between high and low skilled golfers. Whilst overall VE in each group of variables was different between skills groups there were 
no differences between groups in the VE of the separate body position measurements at impact. Looking at Table 2 there was no consistent pattern of higher or lower variability between the groups and therefore the current work provides no support for the hypothesis that golfers of a higher skill level will show less variability. Results from Bradshaw et al. (2009) also suggest this, with no variability differences found between high and low skilled golfers at impact for trunk angle, and both lead wrist and elbow angles.

Horan et al. (2011) have demonstrated that although the male and female swings emerged with different upper body movement strategies during the downswing there was a common trend of the variability of hand and clubhead trajectory sequentially decreasing during the downswing to impact with the ball. Other sports have shown similar patterns of funneling toward impact with Bootsma and van Wieringen (1990) finding that the variation in the direction of a table tennis bat decreases toward the moment of impact with the ball in a forehand drive. This funneling effect in golf has to be attributed to movements of segments of the body. The funneling of movement variability of the golf club has to be initiated within the golfer's body segments during the downswing. The results of this study show that there is a significant reduction in positional variability at impact from proximal to distal segments of the body, moving along the kinetic chain (Hume, Keogh \& Reid, 2005) of the golf swing. From the stance upwards, the segments that initiate the transition into the downswing (i.e., the pelvis [Cheetham et al., 2008]) present the largest amount of VE in position from trial-trial with the shoulders presenting the least.

The higher the skill of a golfer the lower the shot to shot variability in clubhead speed, efficiency of strike, centeredness of strike, angle of attack, club path, and face angle at impact (Betzler et al., 2012). The hands and clubhead positional VE were not analyzed in this study but future research should aim to see if this trend continues across these segments and into the clubhead's position and whether this is the critical feature that distinguishes between skill levels. Horan et al. (2011) have already established that these segments do not differ between male and females but only tested skilled golfers and so their results cannot be extended to other ability levels. If lesser skilled golfers are unable to continue the funneling of variability to the same extent as skilled golfers then golf coaches should ensure that their methods of teaching address this problem. With the emergence of increasing levels of 3D analysis in the sport of golf it may soon be feasible for coaches to monitor and compare a golfer's variability in body segments and control of the clubhead (i.e., stance, pelvis, shoulders, hands and clubhead) against future established tolerance levels that allows variability to be functional. They should also focus their attention to the critical features of performance: the impact factors where the clubhead strikes the ball. Penner (2003) suggested that the position of the clubhead at the top of the backswing and its movement during the transition phase (first 100ms) into the downswing were critical features of the swing to influence impact with the ball. Bradshaw et al. (2009) also suggest that the address, half backswing, and top of the backswing are the instances where consistency of position is critical to performance. It is possible that the transition phase is where the level of variability in the impact factors is set in motion with Cochran and Stobbs' (1968) experiments suggesting that golfers are unable to react after this phase of the swing. 
It appears that with no significant differences observed in the HSG for the alignment relationships coaches should focus on this key area to progress the LSG swing. The stance to pelvis variability is the key alignment relationship to decrease detrimental variability in LSG. This area merits further study of 3D pelvic positions with larger samples to establish whether this is indeed a critical feature of ability levels. As we have previously shown at address (Langdown et al., 2013) there is a reduction of variability from stance up through the pelvis to the shoulders in distance to the ball, alignment relationships between body segments, and pelvis and shoulder tilt. This would suggest that players are able to reduce variability toward the distal end of the kinematic sequence. It is suggested that the need to produce complex movements in distal segments such as the hands and clubhead could justify the need to reduce variability toward the distal segments of the kinematic sequence.

It was not expected that high and low skilled golfers would demonstrate similar VE in their body position at impact. This may be true of all golfers between professional and handicap category 3 , but it is possible that given the nature of the repeated shots made in this study there was a learning effect in the less skilled golfers whereby they were able to settle into a consistent pattern of hitting shots toward the specified target. Golfers in the current study did not see the entire ball flight and it may be that this extrinsic knowledge of results and potential subsequent adaptation to the swing for the next shot may show that differences in variability do exist between highly skilled and less skilled golfers. A recommendation for future work is to explore the VE presented when a player can see the entire ball flight and also on varying slopes and ball lies for repeated trials which promotes a more representative performance setting (Pinder, Davids, Renshaw \& Araújo, 2011) for the golfer.

\section{Conclusion}

This study has shown that no differences exist in variable error of individual body impact position parameters between low and high skilled golfers, although an overall statistical difference was observed across all parameters. A decreasing variable error has been found in distance to ball, alignment, and tilt measurements for those body segments closer to the distal or accuracy end of the golf swing's kinematic sequence, which is consistent with evidence from other target orientated sports (e.g., Bootsma \& Van Wieringen, 1990). Further research is needed to fully elucidate how the reduction of variability toward the distal segments of the body affect the variability of movement at the hands and clubhead and what the levels of tolerance are for variability across the segments of the body. Representative research should focus upon the effects of varying slopes and ball lies on the swing's kinematic variability and consider the impact of a golfer receiving knowledge of the ball flight in this work. Coaches should focus on reducing the detrimental variability in the stance-pelvis alignment relationship with LSG to improve this group's performance. In practical terms, this requires the coaches to create a more consistent impact position through the pelvis with less skilled golfers. Though this may be difficult for coaches to analyze at impact it may be useful to reduce alignment variability at address which could ultimately dictate the backswing and impact positions (Langdown et al., 2013). 


\section{References}

Arend, S., \& Higgins, J. (1976). A strategy for the classification, subjective analysis and observation of human movement. Journal of Human Movement Studies, 2, $36-52$.

Betzler, N.F., Monk, S.A., Wallace, E.S., \& Otto, S.R. (2012). Variability in clubhead presentation characteristics and ball impact location for golfers' drives. Journal of Sports Sciences, 30, 439-448. PubMed doi:10.1080/02640414.2011.653981

Bootsma, R.J., \& Van Wieringen, P.W.C. (1990). Timing an attacking forehand drive in table tennis. Journal of Experimental Psychology. Human Perception and Performance, 16, 21-29. doi:10.1037/0096-1523.16.1.21

Bradshaw, E.J., Keogh, J.W.L., Hume, P.A., Maulder, P.S., Nortje, J., \& Marnewick, M. (2009). The effect of biological movement variability on the performance of the golf swing in high- and low-handicapped players. Research Quarterly for Exercise and Sport, 80, 185-196. PubMed doi:10.1080/02701367.2009.10599552

Cheetham, P.J., Rose, G.A., Hinrichs, R.N., Neal, R.J., Mottram, R.E., Hurrion, P.D., \& Vint, P.F.Crews, D., \& Lutz, R. (Eds.). (2008). Comparison of kinematic sequence parameters between amateur and professional golfers. In D. Crews, \& R. Lutz (Eds.), Science and Golf V: Proceedings of the World Scientific Congress of Golf (pp. 30-36). Mesa: Energy in Motion Inc.

Cochran, A., \& Stobbs, J. (1968). The search for the perfect swing. Philadelphia: Lippincott.

Hebron, M. (2001). Golf Swing Secrets... and Lies: Six Timeless Lessons. New York: Learning Golf Inc.

Horan, S.A., Evans, K., \& Kavanagh, J.J. (2011). Movement variability in the golf swing of male and female skilled golfers. Medicine and Science in Sports and Exercise, 43, 1474-1483. PubMed doi:10.1249/MSS.0b013e318210fe03

Hume, P.A., Keogh, J., \& Reid, D. (2005). The role of biomechanics in maximising distance and accuracy of golf shots. Sports Medicine (Auckland, N.Z.), 35, 429-449. PubMed doi:10.2165/00007256-200535050-00005

Langdown, B.L., Bridge, M., \& Li, F-X. (2012). Variability of movement in the golf swing. Sports Biomechanics, 11, 273-287. PubMed doi:10.1080/14763141.2011. 650187

Langdown, B.L., Bridge, M., \& Li, F-X. (2013).Address position variability in golfers of differing skill level. International Journal of Golf Science, 2, 1-9

Lee, D. N., Lishman, J. R., \& Thomson, J. A. (1982). Regulation of gait in long jumping. Journal of Experimental Psychology: Human Perception and Performance, 8(3), 448.

Mann, R., Griffin, F., \& Yocom, G. (1998). Swing like a pro. New York: Broadway Books.

McPherson, M. (1990). A systematic approach to skill analysis. Sports Science Periodical on Research Technology in Sport, 11(1), 1-10.

Miller, S.A. (2002). Variability in Basketball Shooting: Practical Implications. In Y. Hong (Ed.), International Research in Sports Biomechanics (pp. 27-34). London: Routledge.

Newell, K.M. (1986). Constraints on the development of coordination. In M.G. Wade \& H.T.A. Whiting (Eds.), Motor development in children: Aspects of coordination and control (pp. 341-360). Boston: Martinus Nijhoff.

Penner, A. R. (2003). The physics of golf. Reports on Progress in Physics, 66(2), 131.

Pinder, R.A., Davids, K., Renshaw, I., \& Araújo, D. (2011). Representative Learning Design and Functionality of Research and Practice in Sport. Journal of Sport \& Exercise Psychology, 33, 146-155.

Schmidt, R.A., \& Lee, T.D. (2005). Motor control and learning: A behavioural emphasis. Champaign, IL: Human Kinetics.

Scott, M. A., Li, F. X., \& Davids, K. (1997). Expertise and the regulation of gait in the approach phase of the long jump. Journal of sports sciences, 15(6), 597-605. 
Tuxen, F. (2009). The impact of John Jacobs on golf coaching: A commentary. In S. Jenkins (Ed.), Annual Review of Golf Coaching (pp. 39-49). Multi Science Publishing.

Verbruggen, F., \& Logan, G.D. (2009). Models of response inhibition in the stop-signal and stop-change paradigms. Neuroscience and Biobehavioral Reviews, 33, 647-661. PubMed doi:10.1016/j.neubiorev.2008.08.014 